\title{
Influence of an Advanced Buffer Layer on the Optical Properties of an InGaN/GaN MQW Grown on a (111) Silicon Substrate
}

\author{
D. R. HANG* and M. M. C. CHOU \\ Department of Materials Science and Optoelectronic Engineering, \\ and Center for Nanoscience and Nanotechnology, \\ National Sun Yat-sen University, Kaohsiung 804, Taiwan
}

\section{H. HSIEH}

Institute of Materials Science and Engineering, National Sun Yat-sen University, Kaohsiung 804, Taiwan

\author{
M. Heuken
}

AIXTRON AG, Kackertstrasse 15-17, Aachen, Germany

(Received 6 September 2006)

\begin{abstract}
We study the influence of a buffer layer on the optical properties of InGaN/GaN multiple quantum wells (MQWs) grown on silicon substrates by using metalorganic vapor phase epitaxy. To overcome the large lattice mismatch and the difference in the thermal expansion coefficients by which a high dislocation density occurs, we grow the MQWs on an advanced buffer structure consisting of low-temperature (LT) AlN and a high-temperature (HT) AlN/AlGaN/GaN stack. The Raman spectra confirm that the biaxial tensile strain is reduced by the insertion of the alternating LT and HT buffer layers. Moreover, we find the room-temperature internal quantum efficiency can be improved. Our results suggest that the enhanced optical performance comes from the reduced number of nonradiative recombination centers brought about by the LT and HT composite buffer layers.
\end{abstract}

PACS numbers: 78.66-w, 78.55.Cr, 78.47.+p

Keywords: GaN, InGaN, Quantum well

\section{INTRODUCTION}

GaN-based nitride semiconductors have played important roles in optoelectronic devices such as light-emitting diodes and laser diodes $[1,2]$. Due to the lack of suited homosubstrates, these devices are usually grown on heterosubstrates such as sapphire and SiC. Recently, GaNbased materials grown on silicon substrates have drawn a lot of attention [3]. Silicon offers the advantages of high crystalline quality, large wafer size, and low cost. Moreover, this approach is a promising candidate for integration of GaN-based optoelectronics with Si-based electronics in the future. One of the major challenges for this approach is to overcome the large lattice mismatch and difference in thermal expansion coefficients because of which a high dislocation density occurs. From the device point of view, defects induced by lattice imperfections and residual strain influence their optical performance by a large extent because they can act as nonrecombi-

*Corresponding Author: drhang@mail.nsysu.edu.tw; Tel: +886-7-5252000-4066; Fax: +886-7-5254099 nation centers (NRCs). Considerable efforts have been made to reduce the dislocation density and the residual strain to improve the crystalline quality of GaN samples deposited on silicon substrates. Silicon delta-doping, epitaxial lateral overgrowth, and substrate engineering have been proposed to improve the crystalline growth [4-6].

In this paper, we report an approach based on an advanced buffer structure grown prior to the active layers. The influence of such buffer layers on the optical properties of InGaN/GaN multiple quantum wells (MQWs) grown on silicon substrates is investigated. We show that this approach is able to improve the optical efficiency and that the enhanced optical performance is related to a reduction of the strain and NRCs brought about by the buffer structures.

\section{EXPERIMENT}

The samples used for our study are InGaN/GaN MQWs grown on 2-inch $\mathrm{Si}$ (111) substrates by using metalorganic vapor phase epitaxy. Trimethylgal- 


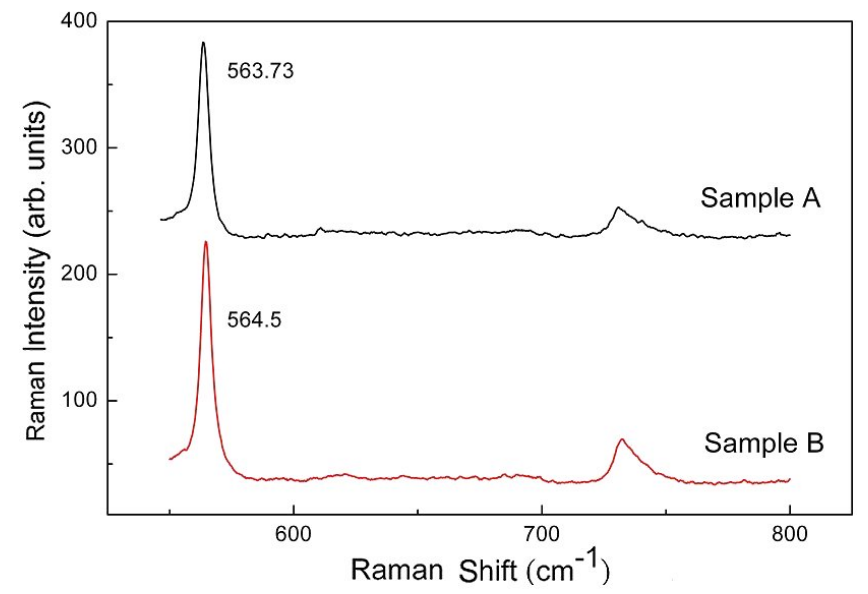

Fig. 1. Room-temperature micro-Raman spectra of our samples.

lium, trimethy-indium, trimethyl-aluminum, ammonia, and SiH4 were used for the growth. The MQWs are grown on an advanced buffer structure consisting of lowtemperature (LT) AlN and a high-temperature (HT) AlN/AlGaN/GaN stack. The LT layer serves to reduce the large strain while the HT layer lessens the difference in thermal expansion. For sample A, a 32-nm LT AlN nucleation layer was grown on the substrate. This was followed by HT 50-nm AlN and 500-nm GaN deposition. Then, a second thin LT AlN nucleation layer and 600-nm-thick HT GaN:Si $\left(n_{\mathrm{Si}} \sim 10^{19} \mathrm{~cm}^{-3}\right)$ were grown on top of the first HT layers. On these buffer structures, the main MQW structure, consisting of tenperiod 2-nm-thick $\mathrm{In}_{0.25} \mathrm{Ga}_{0.75} \mathrm{~N}$ and 4-nm-thick GaN:Si $\left(n_{\mathrm{Si}} \sim 10^{20} \mathrm{~cm}^{-3}\right)$, was grown psudomorphically. Finally, 50-nm GaN were grown as a cap layer. A refined buffer was used for sample B, in which two HT stacks consist of $\mathrm{HT} 100-\mathrm{nm} \mathrm{AlN}$, and $100-\mathrm{nm} \mathrm{Al}_{0.3} \mathrm{Ga}_{0.7} \mathrm{~N}$, and $60-\mathrm{nm} \mathrm{Al}_{0.2} \mathrm{Ga}_{0.8} \mathrm{~N}$ layers were inserted before the start of the growth of each thick GaN layer in the abovementioned structures.

The Raman spectra were measured under 514.5-nm excitation and recorded by using a Jobin-Yvon T64000 micro-Raman system under the $z\left(x_{-}\right) \underline{z}$ backscattering configuration with the scattering light parallel to the $c$ axis of the epilayers. The laser light was focused to a 1$\mu \mathrm{m}$ spot size. For the photoluminescence (PL) measurements, the sample was excited by using a chopped He$\mathrm{Cd}$ laser beam. The emission was collected and sent to a Jobin-Yvon Triax 550 monochromator and detected by a Hamamatsu R928 photomultiplier tube. For the timeresolved photoluminescence (TRPL) measurements, a pulsed diode laser operating at $405 \mathrm{~nm}$ was used as an excitation light source. The signal was analyzed by using a PicoQuant time-correlated single photon counting system. Samples were mounted onto the cold head of a closed cycle refrigerator and cooled down to the desired temperatures for the measurements.
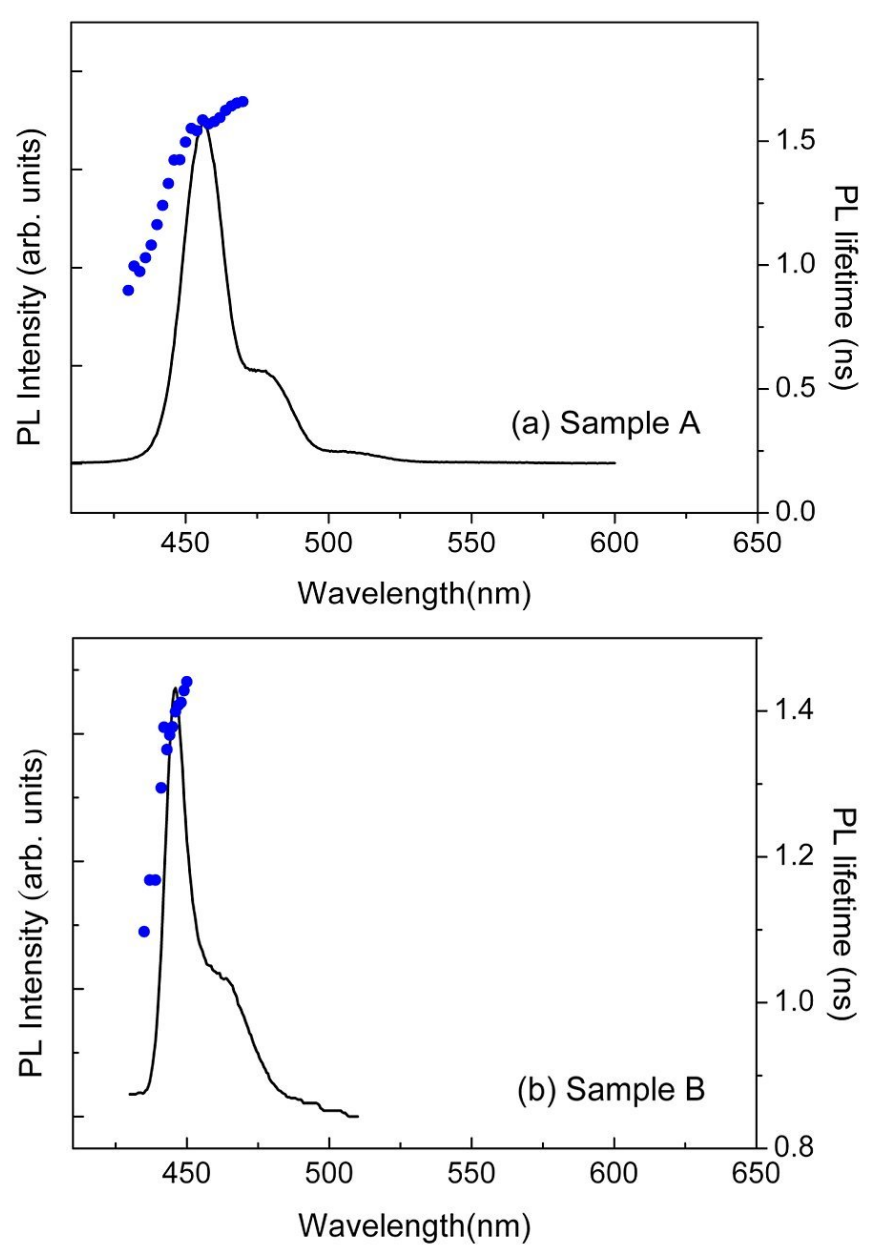

Fig. 2. (a) PL spectra and PL lifetime (closed circles) measured at $10 \mathrm{~K}$ for sample $\mathrm{A}$. (b) The same thing for sample B.

\section{RESULTS AND DISCUSSION}

Fig. 1 shows the micro-Raman spectra of our samples at room temperature. Both the $\mathrm{E}_{2}$-high and $\mathrm{A}_{1}$ $\mathrm{L}_{o}$ modes of $\mathrm{GaN}$ are observed. The $\mathrm{E}_{2}$-high mode for sample $\mathrm{A}$ is $563.73 \mathrm{~cm}^{-1}$ while for sample $\mathrm{B}$, it is shifted to $564.5 \mathrm{~cm}^{-1}$. Compared with the standard value of $567.5 \mathrm{~cm}^{-1}$ for bulk GaN [5,7], the $\mathrm{E}_{2}$-high peaks for both samples show redshifts with respect to that of bulk GaN. Because of the lattice and the thermal mismatch, the GaN epilayer is subjected to a biaxial tensile stress when grown pseudomorphically on silicon (111) substrates. The $\mathrm{E}_{2}$-high mode in the Raman measurements is often used to probe quantitatively the in-plane strain because this mode is found to be sensitive to the biaxial stress in GaN layers [8]. The biaxial stress can be estimated according to the relation $\Delta \omega=C \sigma[9]$, where $\Delta \omega$ is the phonon peak shift, $C$ is the stress coefficient, and $\sigma$ is the biaxial stress. By using the stress coefficient $4.3 \mathrm{~cm}^{-1} / \mathrm{GPa}[3,6,10]$, we found the calculated stress in the underlying GaN layer to be $0.877 \mathrm{GPa}$ for 

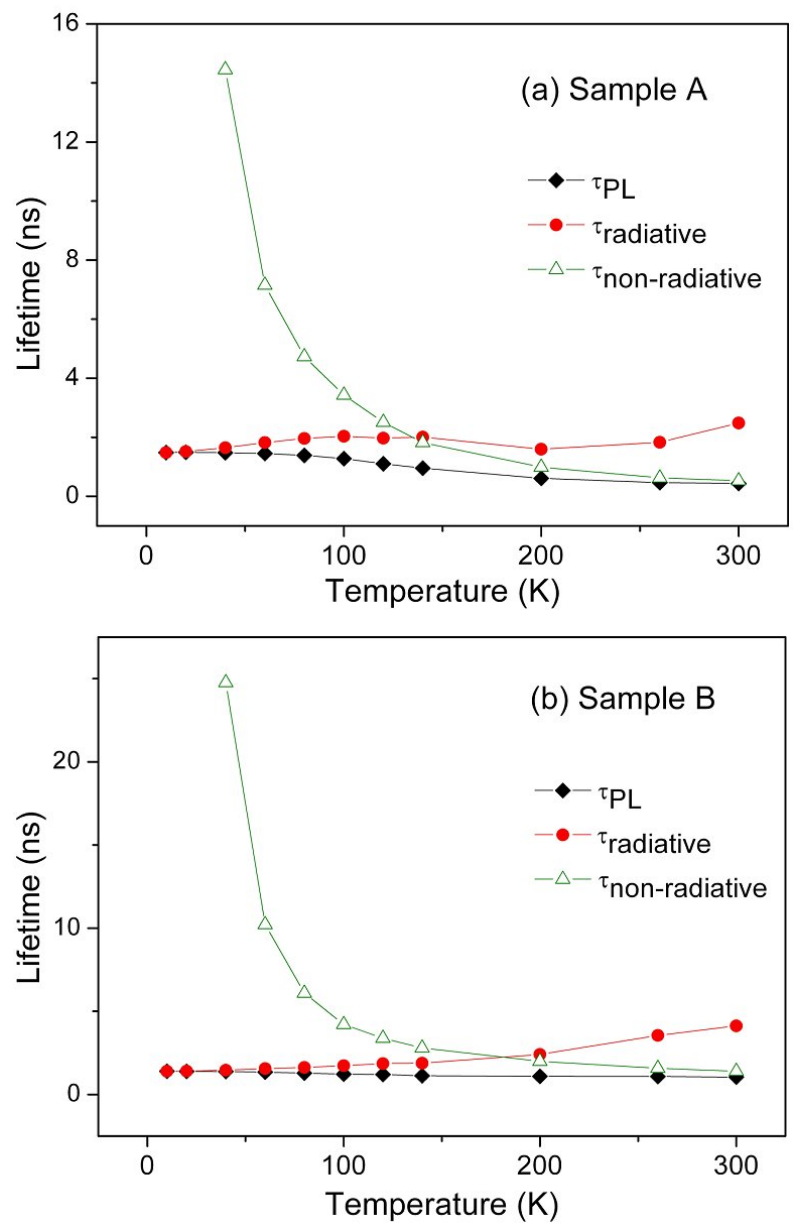

Fig. 3. Temperature dependence of the PL lifetime $\tau_{\mathrm{PL}}$ (closed diamonds), the radiative lifetime $\tau_{\text {rad }}$ (closed circles), and the nonradiative lifetime $\tau_{\text {nonrad }}$ (open triangles) for (a) sample A and (b) sample B.

sample A and $0.698 \mathrm{GPa}$ for sample B. A reduction of tensile stress by an amount of $0.179 \mathrm{GPa}$ is observed in our study. Such an estimate indicates that the insertion of additional HT stacks gives rise to a significant reduction of the in-plane tensile stress in the epilayers, which is beneficial because the crack formation and threading dislocation density can be reduced in this way $[11,12]$.

Figs. 2(a) and (b) display the PL spectra measured at $10 \mathrm{~K}$ for both samples. They show similar band profiles, and the main peak wavelengths are 456 and $446 \mathrm{~nm}$ for samples A and B, respectively. With a similar top quantum well structure, the peak shift can be largely related to the reduced strain in sample B revealed by the Raman observation. To further understand the optical emission of our samples, we performed TRPL measurements. We found that the PL decay, in general, can be fitted with an exponential decay. The emission energy dependence of the PL lifetime, $\tau_{\mathrm{PL}}$, at $10 \mathrm{~K}$ is shown in Fig. 2 as closed circles for both samples. The $\tau_{\mathrm{PL}}$ values are found to increase with decreasing photon energy for both samples, which is a characteristic of a localized system. In addi-

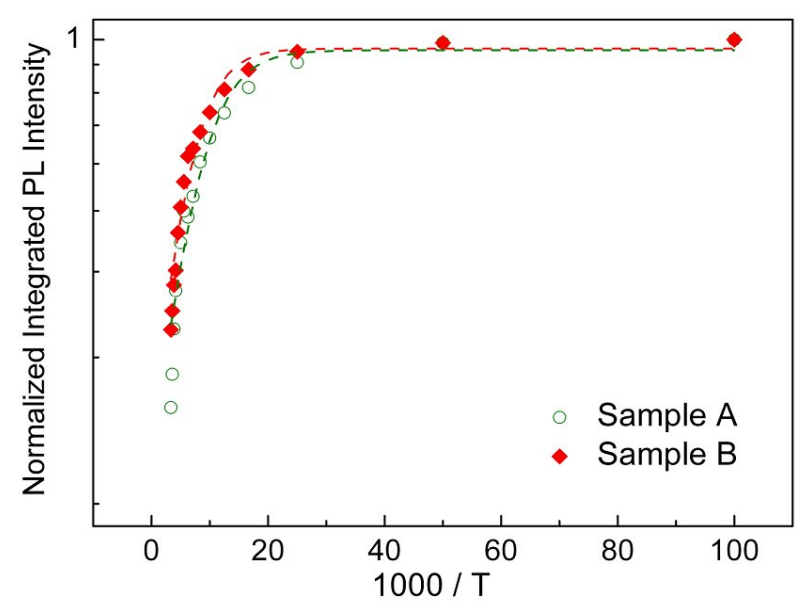

Fig. 4. Temperature dependence of the integrated PL intensity of the main emission band for both samples. The constant $I(T)$ value in the low-temperature range is normalized to unity. The dashed lines are the least-squares fits of the data with Eq. (1).

tion, temperature-dependent TRPL measurements were performed. The temperature dependence of $\tau_{\mathrm{PL}}$ is shown in Figs. 3(a) and (b) as closed diamonds. The decay of excitons consists of both radiative recombination and a transfer to localized states. As the temperature rises to a certain extent, the excitons delocalize with the aid of enhanced thermal energy and recombine via nonradiative centers quickly. Therefore, the lifetime $\tau_{\mathrm{PL}}$ drops, as shown in Figs. 3(a) and (b), for both samples.

The temperature dependence of the integrated PL intensity of the main PL band for our samples is shown in Fig. 4. The general trend is for the integrated PL intensity to stay near unity for temperature lower than $100 \mathrm{~K}$ because the PL is expected to be dominated by localized excitons in this temperature range. The integrated PL intensity then decreases with increasing temperature due to exciton delocalization. We find that the integrated PL intensity for sample A drops more rapidly than that for sample B. Since the nonradiative recombination process is generally frozen at low temperatures, the internal quantum efficiency $\eta_{\text {int }}$ can be set to unity. As the temperature increases, the internal quantum efficiency $\eta_{\text {int }}(T)$ drops due to the enhanced nonradiative recombination. At room temperature, the internal quantum efficiency $\eta_{\text {int }}(R T)$ for sample $\mathrm{A}$ is $17 \%$, while that for sample B is raised to $25 \%$. This efficiency is quite good even compared with those for samples grown on conventional sapphire substrates.

The thermal quenching of the integrated PL intensity was fitted by using [13]

$$
I(T)=I_{0} /\left[1+D \exp \left(-E_{a} / k T\right)\right],
$$

where $I(T)$ is the integrated PL intensity at $T \mathrm{~K}, I_{0}$ is a scaling factor, $D$ is a process rate parameter, $E_{a}$ is the activation energy, and $k$ is the Boltzmann constant. The dashed lines in Fig. 4 are the least-squares fits of data 
with Eq. (1). The fitted values of $E_{a}$ are 20 and $24 \mathrm{meV}$ for sample A and B, respectively.

It is known that the PL lifetime can be decomposed as $1 / \tau_{\mathrm{PL}}(T)=1 / \tau_{\text {rad }}(T)+1 / \tau_{\text {nonrad }}(T)$, where $\tau_{\text {rad }}(T)$ and $\tau_{\text {nonrad }}(T)$ are the radiative and the nonradiative lifetimes at $T \mathrm{~K}$, respectively. On the other hand, the internal quantum efficiency $\eta_{\text {int }}(T)$ is related to the radiative lifetime $\tau_{\text {rad }}(T)$ and the nonradiative lifetime $\tau_{\text {nonrad }}(T)$ by the relation $\eta_{\text {int }}(T)=$ $\tau_{\text {nonrad }}(T) /\left[\tau_{\text {rad }}(T)+\tau_{\text {nonrad }}(T)\right]$. Hence, we can estimate the temperature dependence of the radiative and the nonradiative lifetimes in terms of $\tau_{\mathrm{PL}}(T)$ and $\eta_{\text {int }}(T)$. The extracted radiative and nonradiative lifetimes for both samples are shown in Figs. 3(a) and (b) as closed circles and open triangles, respectively.

At low $T$, the PL lifetime is dominated by $\tau_{\text {rad }}$; above a certain critical temperature $T_{c}$, the dominance is taken over by $\tau_{\text {nonrad }}$. For both samples, $\tau_{\text {rad }}$ can be seen to be nearly constant below $100 \mathrm{~K}$ and to increase almost linearly with $T$ above $150 \mathrm{~K}$, suggesting that the density of states from which the emission originates changes from zero dimension to two dimensions with increasing $T$ [14]. Above $100 \mathrm{~K}$, more-nearly-free excitons are trapped by the activated nonradiative channels, resulting in shorter $\tau_{\text {nonrad }}$. We found the decrease in $\tau_{\text {nonrad }}$ to be more rapid for sample $\mathrm{A}$ than for sample $\mathrm{B}$. Therefore, $T_{c}$ increases from $\sim 140 \mathrm{~K}$ for sample A to $\sim 180 \mathrm{~K}$ for sample B. This is in reasonable agreement with the activation energies of 20 and $24 \mathrm{meV}$ derived from the thermal quenching in Eq. (1). This indicates that the decrease in lifetime is mainly due to an increased influence of a nonrecombination process. At room temperature, the excitons involved in the radiative recombination are delocalized due to thermalization and then trapped by NRCs. The $\tau_{\text {nonrad }}$ is influenced by the defect density, which provides sources for NRCs, and the $\eta_{\text {int }}(R T)$ is determined by the NRCs. Our Raman results indicate that the strain and the dislocation density can be reduced by the proper insertion of HT stacks. The improved $\eta_{\text {int }}(R T)$ for sample $\mathrm{B}$ can be attributed to the reduced defect density and to NRCs originating from dislocations, which is consistent with a recent report [15].

\section{CONCLUSIONS}

In conclusion, the influence of an advanced buffer layer on the optical properties of InGaN/GaN MQWs grown on silicon substrates is studied. From the Raman measurements, it is found that the insertion of proper composite HT stacks effectively relieves the strain. Hence, the strain and the dislocation density can be reduced in this way. In addition, the room-temperature internal quantum efficiency can be improved, which is attributed to the reduced density of NRCs due to the composite buffer structure. Our results suggest that to improve the optical performance of GaN-based devices on silicon, one must reduce the strain that brings about a high dislocation density and a high number of nonrecombination centers.

\section{ACKNOWLEDGMENTS}

This work is partly supported by the National Science Council of the Republic of China under grant nos. NSC 94-2112-M-110-009 and NSC 94-2218-E-110-008. D. R. Hang acknowledges the financial support from Advanced Crystal Optoelectronics Research Center and Aim for the Top University Plan of National Sun Yat-sen University, Taiwan.

\section{REFERENCES}

[1] S. Nakamura, S. Pearton and G. Fasol, The Blue Laser Diode, 2nd ed. (Springer, Berlin, 2000).

[2] D. R. Hang, C. T. Liang, J. R. Juang, T. Y. Huang, W. K. Hung, Y. F. Chen, G. H. Kim, J. H. Lee and J. H. Lee, J. Appl. Phys. 93, 2055 (2003); D. R. Hang, Y. F. Chen and C. F. Huang, Appl. Phys. Lett. 89, 92116 (2006); D. R. Hang, C. H. Chen, Y. F. Chen, H. X. Jiang and J. Y. Lin, J. Appl. Phys. 90, 1887 (2001).

[3] D. G. Zhao, S. J. Xu, M. H. Xie, S. Y. Tong and H. Yang, Appl. Phys. Lett. 83, 677 (2003); C. T. Liang, K. Y. Chen, N. C. Chen, P. H. Chang and C. A. Chang, Appl. Phys. Lett. 89, 132107 (2006).

[4] O. Contreras, F. A. Ponce, J. Christen, A. Dadgar and A. Krost, Appl. Phys. Lett. 81, 4712 (2002).

[5] L. S. Wang, K. Y. Zang, S. Tripathy and S. J. Chua, Appl. Phys. Lett. 85, 5881 (2004).

[6] D. Wang, S. Jia, K. J. Chen, K. M. Lau, Y. Dikme, P. van Gemmern, Y. C. Lin, H. Kalisch, R. H. Jansen and M. Heuken, Appl. Phys. Lett. 97, 56103 (2005).

[7] S. Tripathy, S. J. Chua, P. Chen and Z. L. Miao, J. Appl. Phys. 92, 3503 (2002).

[8] H. Harima, J. Phys.: Condens. Matter 14, R967 (2002).

[9] J. M. Wagner and F. Bechstedt, Appl. Phys. Lett. 77, 346 (2000).

[10] S. Tripathy, S. J. Chua, P. Chen and Z. L. Miao, J. Appl. Phys. 92, 3503 (2002).

[11] E. Feltin, B. Beaumont, M. Laugt, P. de Mierry, P. Vennegues, H. Lahreche, M. Leroux and P. Gibart, Appl. Phys. Lett. 79, 3230 (2001).

[12] A. Dadgar, M. Poschenrieder, A. Reiher, J. Blasing, J. Christen, A. Krtschil, T. Finger, T. Hempel, A. Diez and A. Krost, Appl. Phys. Lett. 82, 28 (2003).

[13] D. S. Jiang, H. Jung and K. Ploog, J. Appl. Phys. 64, 1371 (1988)

[14] J. Feldman, G. Peter, E. Gobel, P. Dawson, K. Moore, C. Foxon and R. Elliot, Phys. Rev. Lett. 59, 2337 (1987).

[15] T. Akasaka, H. Gotoh, T. Saito and T. Makimoto, Appl. Phys. Lett. 85, 3089 (2004). 